\title{
The unique Antarctic atmosphere: implications for adaptive optics
}

\author{
Jon S. Lawrence ${ }^{1}$, Michael C.B. Ashley ${ }^{1}$, Michael G. Burton ${ }^{1}$, James P. \\ Lloyd $^{2}$, and John W.V. Storey ${ }^{1}$ \\ 1 School of Physics, University of New South Wales, Sydney NSW 2052, Australia \\ 2 California Institute of Technology, Pasadena CA 91125, USA
}

\begin{abstract}
The turbulence structure of the atmosphere is the primary limitation to adaptive optics system performance on extremely large telescopes — driving current world-wide site testing campaigns. The potential for adaptive optics correction on extremely large telescopes located at Dome C station on the Antarctic plateau is investigated here. Due to the unique atmospheric characteristics of this site it is found that a natural guide star adaptive optics system with a single deformable mirror should outperform a multi-conjugate multi-laser guide star system at a mid-latitude site.
\end{abstract}

\section{Introduction}

Data on the turbulence structure of the South Pole $\left(90^{\circ} \mathrm{S}, 2835 \mathrm{~m}\right.$ elevation) atmosphere has been obtained from a number of instruments. A differential image motion monitor [1], a Doppler SODAR (sound and ranging) [2], and a series of microthermal balloon launches [3] have shown that although the average ground level seeing is only mediocre ( 1.8 arcsec) compared with good quality mid-latitude sites such as Mauna Kea $(0.5$ arcsec $)$, the majority of turbulence is confined to within only $300 \mathrm{~m}$ of ground level. The lack of high-altitude turbulence and wind speeds results in an isoplanatic angle and an atmospheric coherence time that is significantly larger than those found at typical mid-latitude sites. These factors offer significant advantages for a South Pole adaptive optics system.

The local topography at sites higher on the Antarctic plateau, such as Dome $\mathrm{C}\left(123^{\circ} \mathrm{E}, 75^{\circ} \mathrm{S}, 3260 \mathrm{~m}\right.$ elevation), promises a lower surface boundary layer and lower wind speeds at all altitudes [4]. This should result in a lower integrated seeing, a larger isoplanatic angle and a longer atmospheric coherence time, further improving the performance of an adaptive optics system.

Data obtained so far from Dome C confirms these expectations. Summertime DIMM measurements of the integrated turbulence has shown a median seeing of 1.1 arcsec (at 0.5 micron) with a strong diurnal variation and periods of very low and stable values [5]. The first winter-time SODAR results [6] confirm a reduction in the boundary layer height (to less than $200 \mathrm{~m}$ ), and a reduction in the strength of turbulence confined within this boundary layer.

More winter-time data is needed to confirm these preliminary results. The high-altitude turbulence will be determined with a Multi-Aperture Scintillation Sensor (MASS) to be deployed at Dome C over winter 2004 as part of 
the AASTINO remote observatory. In 2005, the first planned winter-over season for the station, the turbulence will be completely characterised by a crosscalibration of MASS, SODAR, DIMM, and microthermal measurements (both balloon-borne and tower-mounted). While it will be several years before a complete evaluation of the turbulence conditions at Dome $\mathrm{C}$ is obtained, a number of factors can be analysed now to determine the potential of this site.

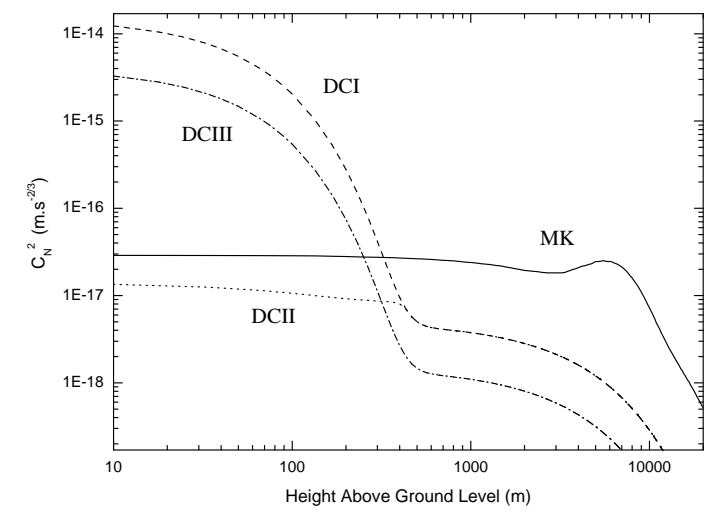

Fig. 1. Refractive index structure constant profiles for Mauna Kea (MK) compared with the three model atmospheres for Dome C (DCI, DCII, and DCIII).

\section{Turbulence Profiles}

Figure 1 shows the refractive index structure constant, $C_{N}^{2}$, profile for Mauna Kea [7]. In order to examine the potential for adaptive optics correction at the Dome C site three different models are developed. Model DCI represents worse case assumptions - the high altitude $(>1000 \mathrm{~m})$ component is set equal to the median South Pole high altitude profile (from microthermal measurements) after translation downwards by the difference in ground elevation $(425 \mathrm{~m})$. The surface boundary layer turbulence contribution for this model is scaled to give an integrated seeing equal to the median summer-time value (1.1 $\operatorname{arcsec})$. SODAR winter-time measurements from Dome $\mathrm{C}$ show that the turbulence within the first $900 \mathrm{~m}$ of the surface is lower than the detection threshold of this instrument for the majority of the time. While there are some uncertainties in these results, they can be used as a lower bound to the expected turbulence conditions. In Model DCII the high altitude component is set equal to the DCI model but the boundary layer is chosen as the noise threshold of the SODAR instrument this gives an integrated seeing of 0.12 arcsec. In order to investigate the effects for a range of conditions a third model is developed (DCIII) representing a larger 
isoplanatic angle (30 arcsec) and an integrated seeing equivalent to the Mauna Kea model.

The wind speed for the Mauna Kea atmosphere is based on the standard Bufton model [8], with a ground wind speed of $10 \mathrm{~m} \cdot \mathrm{s}^{-1}$, and a peak of $35 \mathrm{~m} \cdot \mathrm{s}^{-1}$ at the $10 \mathrm{~km}$ tropopause layer. For the Dome $\mathrm{C}$ models the ground wind speed is set to $2.5 \mathrm{~m} . \mathrm{s}^{-1}$, with a peak of $10 \mathrm{~m} . \mathrm{s}^{-1}$ at the $7 \mathrm{~km}$ tropopause layer. These wind speeds are consistent with summer time radiosonde measurements.

Atmospheric parameters derived from these models are shown in Table 1.

Table 1. Atmospheric parameters at $500 \mathrm{~nm}$ derived from model profiles of wind speed and refractive index structure constant

\begin{tabular}{lllll}
\hline Parameter & Mauna Kea & Dome C I & Dome C II & Dome C III \\
\hline$r_{0}(\mathrm{~m})$ & 0.20 & 0.09 & 0.85 & 0.20 \\
seeing (arcsec) & 0.50 & 1.10 & 0.12 & 0.50 \\
isoplanatic angle (arcsec) & 2.0 & 13.3 & 13.6 & 30.0 \\
Greenwood frequency $(\mathrm{Hz})$ & 50 & 12 & 2 & 5 \\
Tyler frequency $(\mathrm{Hz})$ & 0.77 & 0.16 & 0.04 & 0.08 \\
\hline
\end{tabular}

\section{Adaptive Optics Performance}

Table 2 shows an error budget (rms nm wavefront errors introduced in the adaptive optics correction) for a natural-guide-star adaptive optics system with a single-deformable-mirror on a $30 \mathrm{~m}$ telescope. Errors for the Mauna Kea model are from [7]. For each of the Dome $\mathrm{C}$ atmospheric models the sub-aperture diameter, $d_{s}$, high-order feedback loop bandwidth, $f_{h}$, tip-tilt feedback loop bandwidth, $f_{t}, \mathrm{~K}$ band guide star magnitude, $m_{k}$, and the angular separation between guide star and object star, $\theta$, have been optimised to give the minimum error corresponding to a particular sky coverage factor (matching the 15 arcsec separation and 14.8 guide star magnitude of the Mauna Kea budget). Wavefront errors are determined following the formalism of Hardy [8], under approximately equivalent conditions (wavefront sensor wavelength, feedback loop parameters) to the CELT error budget in [7].

For each of the Dome $\mathrm{C}$ atmospheres the significantly longer atmospheric coherence time (i.e., lower Greenwood frequency) allows a reduced feedback loop bandwidth and sampling frequency; this decreases both the bandwidth error and signal-to-noise ratio error. Additionally, the larger isoplanatic angles of the Dome $\mathrm{C}$ models decrease the anisoplanatic error and allow larger separation angles to be used. This allows brighter guide stars to be used (also reducing the signalto-noise ratio error) and allows a smaller sub-aperture (decreasing the fitting error). 
Table 2. Error budget: wavefront errors in $\mathrm{nm}$ rms for a single natural guide star single deformable mirror adaptive optics system on a $30 \mathrm{~m}$ telescope at Mauna Kea compared with three Dome C models. Each case represents the same sky coverage factor.

\begin{tabular}{lllll}
\hline error terms & Mauna Kea $^{\mathrm{a}}$ & Dome C I $^{\mathrm{b}}$ & Dome C II $^{\mathrm{c}}$ & Dome C III $^{\mathrm{d}}$ \\
\hline anisoplanatism & 319 & 157 & 75 & 78 \\
signal-to-noise & 226 & 103 & 35 & 45 \\
bandwidth & 195 & 103 & 34.7 & 56 \\
fitting & 193 & 104 & 37 & 46 \\
tilt signal & 40 & 32 & 10 & 14 \\
tilt bandwidth & 70 & 20 & 12 & 10 \\
tilt anisoplanatism & 75 & 55 & 21 & 27 \\
instrument and mirror & 90 & 90 & 90 & 90 \\
\hline Total & $500(375)$ & $262(203)$ & $134(110)$ & $150(125)$ \\
\hline
\end{tabular}

${ }^{\mathrm{a}} \theta=15 \operatorname{arcsec}, m_{k}=14.8, f_{h}=6.7 \mathrm{~Hz}, f_{t}=8.4 \mathrm{~Hz}, d_{s}=1.36 \mathrm{~m}$.

b $\theta=40 \operatorname{arcsec}, m_{k}=12.2, f_{h}=3.4 \mathrm{~Hz}, f_{t}=4.8 \mathrm{~Hz}, d_{s}=0.30 \mathrm{~m}$.

c $\theta=15 \operatorname{arcsec}, m_{k}=14.8, f_{h}=2.2 \mathrm{~Hz}, f_{t}=3.6 \mathrm{~Hz}, d_{s}=0.80 \mathrm{~m}$.

${ }^{\mathrm{d}} \theta=40 \operatorname{arcsec}, m_{k}=12.2, f_{h}=3.2 \mathrm{~Hz}, f_{t}=4.8 \mathrm{~Hz}, d_{s}=0.25 \mathrm{~m}$.

$\mathrm{d}$ error in brackets is on-axis.

For the worst case Dome C model, a total error of $260 \mathrm{~nm}$ rms should be achievable. This is almost equivalent to the floor requirement for an multiconjugate adaptive optics system on CELT at Mauna Kea (248 nm rms [7]) consisting of several deformable mirrors and several laser guide stars.

The DCII and DCIII models allow correction to similar values (133 and $150 \mathrm{~nm}$ respectively). This approaches the goal for an MCAO system on CELT at Mauna Kea of $133 \mathrm{~nm}$ [7]. This level of correction at Mauna Kea would require at least 9 sodium laser guide stars (with uplink), 4 deformable mirrors and 20000 actuators.

Table 2 also shows the on-axis error for each atmosphere (i.e., ignoring the high-order and tilt anisoplanatic errors). The on-axis performance of the Mauna Kea telescope is a significant improvement due to the large anisoplanatic contribution. For the best Dome $\mathrm{C}$ conditions removing the off-axis errors results in a total error which is limited primarily by the uncorrected instrument and telescope terms. If this term can be reduced by calibration the primary limitation is then the fitting error. This demonstrates the possibility for extreme adaptive optics with such an atmosphere. By using brighter guide stars, and including more actuators, very low wavefront errors leading to high Strehl ratios in the visible becomes feasible.

The field-of-view of an adaptive optics system can be dramatically increased by the use of laser guide stars. The degree of correction is ultimately determined, 
however, by the residual tip-tilt errors, as the tip-tilt terms must be corrected with natural guide stars. Although the Dome C natural guide star system performs as well as the laser guide system at a mid-latitude site in terms of residual error, the low tip-tilt errors of the Dome $\mathrm{C}$ atmosphere indicate that the implementation of laser guide stars at such a site should result in an even further increase in field-of-view and reduction in wavefront error.
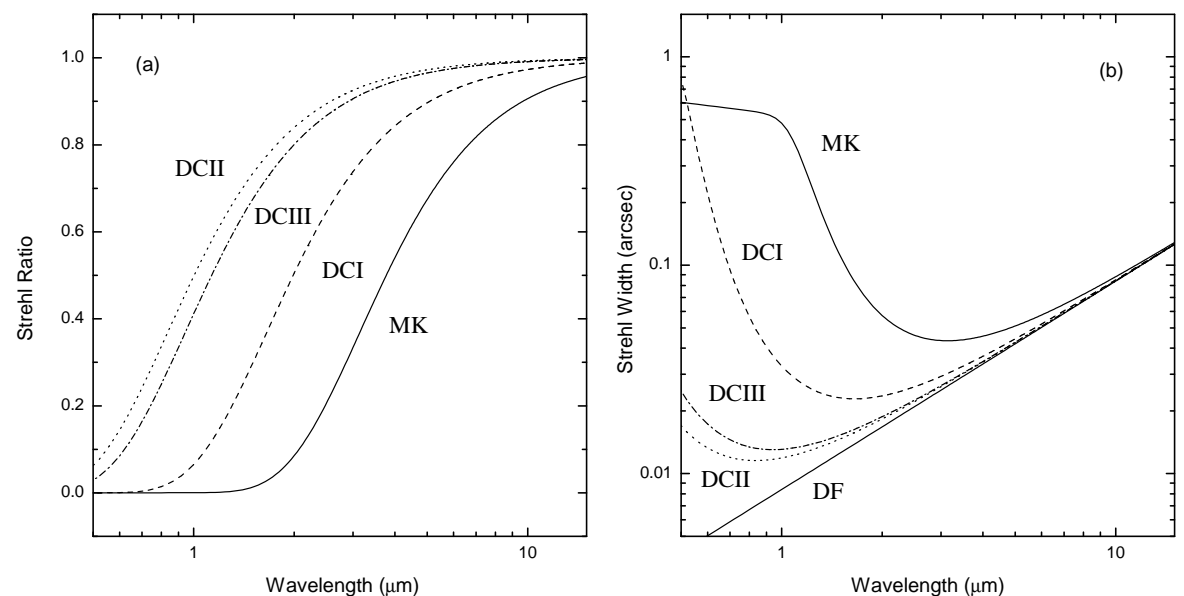

Fig. 2. Strehl ratio (a) and Strehl width (b) versus wavelength for MK, DCI, DCII, and DCIII with natural guide star adaptive optics system. Diffraction-limit (DF) for a $30 \mathrm{~m}$ telescope is also shown in (b).

The Strehl ratio and Strehl width for off-axis correction is shown for each atmosphere in Figures 2 (a) and (b) respectively. For the most pessimistic Dome C model the improvements are substantial and performance is such that diffractionlimited observations over a significant percentage of the sky can be achieved longward of about $3 \mu \mathrm{m}$ compared to $7 \mu \mathrm{m}$ at Manua Kea. If the more optimistic Dome $\mathrm{C}$ models apply, visible light adaptive optics becomes possible with the natural guide star system and a single deformable mirror. Diffraction limit is achieved at $2 \mu \mathrm{m}$ and a Strehl ratio of 0.1 is achieved at $600 \mathrm{~nm}$. Further reduction in the sky coverage factor allows even better correction in the visible as brighter guide stars can be used.

The turbulence and wind speed profiles for the Dome $\mathrm{C}$ atmospheres described here are highly speculative, and the true conditions may vary significantly. This quantification of the possibilities, however, demonstrates the potential of this site.

Although only turbulence conditions have been discussed here, many other characteristics must be considered for a complete site evaluation. The lower emission and higher transmission of the Dome $\mathrm{C}$ atmosphere indicates that infrared 
point source sensitivity should be up to an order of magnitude better than at any mid-latitude site [9]. This is combined with an increase in sensitivity of the Dome $\mathrm{C}$ telescope due to the adaptive optics system performance - fewer warm optical elements are needed for the same resolution or equivalently, increased resolution is obtained with the same number of optical elements. Additional factors, such as the low average and maximum ground wind speeds, and the lack of seismic activity, offer structural advantages to any large Dome C telescope. The ramifications to telescope design of the extreme low temperature environment and the necessity of mounting the telescope on ice, still need to be addressed.

\section{Conclusion}

There is much current interest in the search for the most appropriate sites for the next generation of extremely large telescopes. Adaptive optics error budgets presented here demonstrate the potential offered by one site, Dome $\mathrm{C}$, located on the Antarctic plateau. The benefits arise due to the unique lack of high altitude turbulence and wind encountered at sites on the Antarctic plateau. While the results here are based on preliminary data, the advantages offered over midlatitude sites deserve serious consideration.

\section{References}

1. T. Travouillon, M.C.B. Ashley, M.G. Burton, J.W.V. Storey, P. Conroy, G. Hovey, M. Jarnyk, R. Sutherland, R.F. Loewenstein: Astron. Astrophys. 409, 1169 (2003)

2. T.Travouillon, M.C.B. Ashey, M.G. Burton, J.W.V. Storey, R.F. Lowenstein: Astron. Astrophys. 400, 1163 (2002)

3. R.D. Marks, J.Vernin, M.Azouit, J.W. Briggs, M.G. Burton, M.C.B. Ashley, J.F. Manigault: Astron. Astrophys. Suppl. Ser. 118, 385 (1996)

4. R.D. Marks: Astron. Astrophys. 385, 328 (2002).

5. E. Aristidi, A. Agabi, J. Vernin, M. Azouit, F. Martin, A. Ziad, E. Fossat: Astron. Astrophys. 406, L19 (2003)

6. T. Travouillon, M.C.B. Ashley, M.G. Burton, J.S. Lawrence, J.W.V. Storey: 'Low atmosphere turbulence at Dome C: preliminary results'. In: The scientific outlook for Astronomy and Astrophysics Research at the CONCORDIA station, Anacapri, Italy, April, 2003, Mem. S.A. It. in press

7. R. Dekany, B. Bauman, D. Gavel, M. Troy, B. Macintosh, M. Britton: 'Initial concepts for CELT adaptive optics'. In: Adaptive optics System Technologies II ed. by P.L. Wizinowich, D. Bonaccini, (Proc. SPIE vol. 4839 2003)

8. J.W. Hardy, Adaptive optics for astronomical telescopes. (Oxford University Press, New York 1998)

9. J.S. Lawrence: Publ. Astron. Soc. Pac. submitted (2003) 